\title{
Targeting the Supply Lines of Cancer - A Possible Strategy for Combating the Disease?
}

\author{
OLIVER ABRAHAMSEN, EVA BALSLEV and ESTRID HØGDALL \\ Department of Pathology, Molecular Unit, Herlev Hospital, \\ University of Copenhagen, Copenhagen, Denmark
}

\begin{abstract}
Cancer cells alter and up-regulate their metabolic activity in order to facilitate the increased demands of malignancy. This leads to an increased need for metabolic "building materials", for example glucose and amino acids. The blood circulation represents the principal supply lines delivering these materials. It, therefore, becomes relevant to investigate if these supply lines - in terms of the concentrations of building materials in the blood - may exhibit a therapeutic window and could be intervened, as they deliver the most basal components required to exert malignant functioning. A key aspect in this strategy is that it targets - in theory - the thermodynamic foundation enabling the activities that, essentially, "make a cancer a cancer". As an initial step, this review examines if the metabolic supply lines carry clinical implications; specifically, if they impact survival and the development of metastases in patients with cancer. Furthermore, it presents and discusses perspectives on potentially targeting these supply lines.
\end{abstract}

Despite extensive efforts, cancer remains one of the leading causes of death worldwide. Approximately 9.6 million people died from cancer in 2018 (1). Developing new and improved treatment strategies to combat the disease is, therefore, of paramount importance.

Cancer cells, contrary to normal cells, are characterized by uncontrolled proliferation and being invasive and metastasizing. Cancer cells accordingly alter and up-regulate

This article is freely accessible online.

Correspondence to: Professor Estrid Høgdall, Department of Pathology, Molecular Unit, Herlev University Hospital, Borgmester Ib Juuls Vej 25, 2730 Herlev, Denmark. Tel: +45 38689132, e-mail: estrid.hoegdall@regionh.dk

Key Words: Cancer, metabolism, survival, amino acids, glucose, review. their metabolic activity in order to facilitate these increased demands of malignancy. Thus, an adequate bioenergetic and biosynthetic foundation is needed to support for instance the rapid cell proliferation $(2,3)$. An increase in metabolic activity is followed by an increased utilization and thereby need for metabolic "building materials", for example glucose and amino acids. The blood circulation represents the principal supply lines delivering these materials. Therefore, it can be speculated that a higher circulatory supply may provide advantageous circumstances for the cancer to develop, and likewise, that a limited supply could potentially compromise progression of disease. On this basis, it becomes relevant to investigate if these supply lines, potentially representing a thermodynamically anchored necessity for the malignant activity, could be targeted. The aim would be to investigate if there exists a therapeutic window for a given pattern of the supply lines - in terms of the concentrations of these "building materials" in the blood - where the activity of normal cells would be maintained, but that of the malignant cells would be compromised. A key aspect in this strategy is that it targets - in theory - the fundamental thermodynamic foundation that enables the activities that, in essence, "make a cancer a cancer".

Generally, the metabolic reprogramming occurring in cancer cells is comprehensive and involves numerous metabolic pathways and mechanisms, and these changes are associated with complex oncogenic cellular signalling $(4,5)$. However, for investigating the significance of the aforementioned metabolic supply lines, a narrower focus appears suitable. Central to these metabolic alterations are the ones concerning the use of glucose and amino acids (6), and an initial approach could, therefore, be to investigate the significance of the blood concentrations of these. Thus, this review examines if the blood concentrations of glucose and amino acids impact patient survival and the development of metastases in patients diagnosed with cancer. Furthermore, perspectives on potentially targeting these metabolic supply lines are presented and discussed. 


\section{Cancer Metabolism - Illustrating Malignant Utilization of the Supply Lines of Glucose and Amino Acids}

A cornerstone in cancer metabolism is the Warburg effect. The Warburg effect comprises that cancer cells, even when oxygen is sufficiently available, preferentially take up glucose and produce lactate through glycolysis. This rendering allows for a high glycolytic flux which in turn allows for the generation of glycolytic intermediates that are subsequently directed to branching pathways in order to meet the requirements of proliferating cells (7). The first pathway branching off from glycolysis is the pentose phosphate pathway (PPP) where glucose-6-phosphate is used to generate NADPH and ribose-5-phosphate. NADPH is, for instance, needed as reducing equivalent since many biosynthetic reactions are reductive, whereas ribose-5phosphate is a structural component of nucleotides. Likewise, glycerol-3-phosphate is through dihydroxyacetone phosphate (DHAP) made from glyceraldehyde-3-phosphate and is used in the production of phospholipids and hence cellular membranes. These processes illustrate how an increased uptake and utilization of glucose may enable the acquiring of the precursors needed to exert the high proliferation rate and growth intrinsic to cancer (6).

Amino acids are substrates for protein synthesis and perform multiple diverse functions in various processes. Glutamine can act as an important anaplerotic substrate for the tricarboxylic acid (TCA) cycle and is furthermore involved in the biosynthesis of nucleotides and other amino acids (8). In addition to the biosynthetic advantages of the Warburg effect, intermediates of the TCA cycle also function as precursors that are necessary to support proliferation, and use of precursors derived from the TCA cycle is dependent on anaplerotic fluxes that refill the cycle. An anaplerotic pathway can be represented by the carboxylation of pyruvate to form oxaloacetate, but also through acquisition of glutamine. Glutamine can be converted into glutamate which is then converted into $\alpha$-ketoglutarate entering the cycle (9). In line with this, proliferating cells can be observed to be in need of a continuous supply of glutamine to maintain TCA cycle intermediates (10).

A central aspect of malignancy where altered metabolism, in relation to redox balance, plays a role is in the development of metastases. Generally, cancer cells are exposed to increased levels of reactive oxygen species (ROS), and while this to a certain degree is involved in activating transcription factors promoting oncogenesis, too high levels of ROS will inflict cell damage and cell death. Thus, it is critical for cancer cells to promote antioxidant capacity to counter such high ROS levels. Many antioxidant defense systems require NADPH for which one important source is the pentose phosphate pathway. Cancer cells undergoing the processes of metastasis are subjected to significant ROS levels, and upregulating NADPH-generating pathways serves as one of the mechanisms allowing distant metastasis to occur $(3,11)$.

These examples illustrate how metabolic alterations - and the supply lines delivering the basic "building materials" may underlie activities that constitute the essence of malignancy, for instance proliferation and metastasis.

\section{The Clinical Implications: The Impact of the Metabolic Supply Lines on Patient Survival and on the Development of Metastases}

A meta-analysis comprising 12 studies with a total of nearly 10,000 cancer patients showed that hyperglycemia was associated with both shorter overall survival and disease-free survival (12). In concordance, a French study followed 301,948 participants (primary care volunteers) who underwent a health examination between January 1991 and December 2008. Blood glucose measurements were included in the examinations and were afterwards compared to the risk of following cancerrelated death. The study found that higher blood glucose concentrations could be seen to significantly associate with higher rates of cancer-related deaths, whereas no such association was found among participants presenting normoglycemic levels (13). Furthermore, these associations can also be observed at elevated blood glucose concentrations that are still below the diabetic range (12-14). Similar findings are also reported in other relatively large studies (15-17). However, some studies fail to demonstrate a significant association (18) or for instance propose a U-shaped risk trajectory for the concentrations (19). Still, the majority indicate that blood glucose concentration may impact survival. Regarding the development of metastases, one study investigated 1,261 women diagnosed with breast cancer and reported that higher peridiagnostic blood glucose levels correlated with a higher risk of distant metastasis (20). Another study similarly found an increased risk based on higher glucose concentrations in men diagnosed with prostate cancer (21). In contrast, a study including 391 colorectal cancer patients showed no significant correlation to distant metastasis, although glucose levels did appear to affect local tumor malignancy (22).

Higher levels of glucose in the blood could potentially support an effective fueling of the Warburg effect, thus providing the biosynthetic intermediates required for rapid proliferation and enabling ROS-mitigating systems needed for metastasis. An ensuing aspect concerns the increased production of lactate resulting from the Warburg effect, as this production can promote angiogenesis and cell migration and compromise immune responses (23).

When evaluating the studies involving measurements of amino acid concentrations, the interpretation of the results is slightly more complex. A review addressing the alterations 
of plasma-free amino acid profiles in patients with cancer presents the general interpretation that increased usage of amino acids in cancer cells lead to a lower concentration in plasma, although maintaining that some studies nevertheless find higher concentrations to correlate with malignancy, and that type of cancer, disease stage, patient characteristics, measurement technique as well as multiple other factors may influence the result (24).

A study including 93 patients with lung cancer found an association between short survival and decreased levels of serum glutamine, valine and glycine and an increased level of glutamate. The authors reported that the decreased levels might be indicative of a greater consumption by tumors in patients with short survival, and likewise, that the increased glutamate level concordantly reflects the uptake and conversion of glutamine (25). Another study investigated the significance of pretreatment serum glutamine level in 123 patients with colorectal cancer and found that lower levels independently predicted both shorter overall and progressionfree survival (26).

Glutamine plays an important role with its involvement in fueling the TCA cycle and in biosynthetic activities, which may explain why a higher utilization is found to correlate with worse outcomes. Furthermore, both glutamine and glycine are involved in core functions like the production of nucleotides, and, when glutamine is converted to glutamate, in synthesizing the antioxidant glutathione (GSH) which is a key regulator in controlling redox balance (27).

A study investigating preoperative plasma samples from 51 patients with esophageal squamous cell carcinoma compared plasma amino acid concentrations to clinicopathological characteristics. It was found that lymph node metastasis, worse tumor differentiation and clinical stage were significantly associated with decreased levels of multiple amino acids including serine, glycine, asparagine, glutamate, alanine and tyrosine (28).

Serine and glycine are implicated in fueling the important one-carbon metabolism which facilitates the generation of one-carbon units (methyl groups), that can subsequently be used in for instance nucleotide synthesis and other critical processes. One-carbon units can be derived from serine through its hydroxymethyltransferase-mediated (SHMT1/ SHMT2) conversion to glycine, and from glycine through the glycine cleavage system (GCS) $(8,29)$. One-carbon metabolism can also contribute to redox balance and thus may play a role in metastasis $(11,27)$, which could reflect the association between decreased levels and lymph node metastasis reported in the esophageal squamous cell carcinoma study (28).

One-carbon metabolism is furthermore involved in methylation reactions. Tumors frequently display alterations in DNA methylation, for example in the form of hypermethylation of tumor suppressor gene promotors resulting in decreased expression. In this process, SAM (S-adenosylmethionine) operates as a universal methyl donor and is generated from the addition of ATP-derived adenosine to methionine, the recycling of which can again be supported by serine/glycine metabolism (29). Correspondingly, studies indicate that plasma concentration and utilization of methionine may exert an effect on survival, for instance in patients with glioblastoma (30) and head and neck cancers (31).

A study including 197 breast cancer patients compared serum metabolic differences between patients with localized disease and patients with metastases. The study found that patients in the latter group displayed metabolic alterations reflecting the high-energy needs coupled to the aggressive tumor growth in metastatic disease (32).

Another study including patients with papillary thyroid carcinoma also described a serum metabolic signature coupled to the occurrence of distant metastasis, in which for example pathways of alanine, aspartate and glutamate metabolism were of particular importance (33). In a prostate cancer study, corresponding serum and tissue samples were collected from patients at different disease stages, consequently comprising both non-metastatic and metastatic cases. This collection allowed for metabolic profiling at different stages and for the investigation of the metabolic interplay between the circulation and malignant tissues. Several amino acids in serum displayed a downtrend (illustrating increased utilization) during the progression towards more advanced and metastatic disease. In addition, analyses of serum and tissue samples indicated an increased consumption and usage of energy metabolites along disease progression, thereby demonstrating the augmented energy expenditure (34). Thus, studies linking alterations in utilization of glucose and amino acids with the development of metastases can be found, furthermore in studies concerning patients with colorectal cancer (35), renal cell carcinoma (36) and again esophageal squamous cell carcinoma (37).

In this esophageal squamous cell carcinoma study, elevated lactate levels are reported as an important factor during progression, as it may lead to acidification of the microenvironment resulting in matrix degradation, invasiveness and metastatic progression (37).

Another general mechanism concerns the detachment of cancer cells from the extracellular matrix (ECM), as this detachment involves antagonizing effects on the energy metabolism materializing in ATP deficiency (38). A suggested mechanism by which this can be circumvented is through upregulating the TCA cycle to restore the energy levels (11). In the prostate cancer study, the tissue citrate level is seen to decrease during progression, indicating that citrate is oxidized in the TCA cycle to ultimately generate ATP (34) which could propose the employment of such a mechanism. And generally, the TCA cycle can be fueled through utilization of anaplerotic supply lines. 
Table I. Overview of highlighted publications potentially indicating clinical impact of metabolic supply lines.

\begin{tabular}{|c|c|c|c|}
\hline Ref. & $\begin{array}{l}\text { Number of } \\
\text { participants }\end{array}$ & $\begin{array}{l}\text { Type of cancer } \\
\text { investigated }\end{array}$ & $\begin{array}{l}\text { Extracted } \\
\text { key points }\end{array}$ \\
\hline Barua et al. (12) & 9,872 & $\begin{array}{l}\text { Solid tumors, } \\
\text { different types }\end{array}$ & $\begin{array}{l}\text { Higher blood glucose levels associated with shorter } \\
\text { overall survival and disease-free survival }\end{array}$ \\
\hline Simon et al. (13) & 301,948 & Different types & $\begin{array}{l}\text { Higher blood glucose levels associated with } \\
\text { higher rates of cancer-related deaths }\end{array}$ \\
\hline Contiero et al. (20) & 1,261 & Breast cancer & $\begin{array}{l}\text { Higher peridiagnostic blood glucose levels correlated } \\
\text { with higher risk of distant metastasis occurring }\end{array}$ \\
\hline Berker et al. (25) & 93 & Lung cancer & $\begin{array}{l}\text { Lower blood levels of glutamine, valine and glycine and } \\
\text { a higher level of glutamate associated with short survival* }\end{array}$ \\
\hline Ling et al. (26) & 123 & Colorectal cancer & $\begin{array}{l}\text { Lower blood levels of glutamine independently predicted } \\
\text { shorter overall survival and progression-free survival* }\end{array}$ \\
\hline Ma et al. (28) & 51 & $\begin{array}{l}\text { Esophageal squamous } \\
\text { cell carcinoma }\end{array}$ & $\begin{array}{l}\text { Decreased levels of multiple amino acids associated } \\
\text { with lymph node metastasis, worse tumor } \\
\text { differentiation and clinical stage* }\end{array}$ \\
\hline Jobard et al. (32) & 197 & Breast cancer & $\begin{array}{l}\text { Metabolic alterations reflected high-energy needs coupled } \\
\text { to aggressive tumor progression in metastatic disease }\end{array}$ \\
\hline Zheng et al. (34) & 76 & Prostate cancer & $\begin{array}{l}\text { Multiple amino acid levels displayed a downtrend towards } \\
\text { more advanced disease stages. Furthermore, increased } \\
\text { usage of energy metabolites at more advanced disease } \\
\text { stages was observed* }\end{array}$ \\
\hline
\end{tabular}

*Lower levels of amino acids in the blood are interpreted as indicative of increased utilization by malignant cells.

However, not all studies find that amino acid alterations consistently lead to an extensive clinical manifestation. A study investigating the serum metabolic differences between early- and advanced-stage hepatocellular carcinoma patients found alterations in both glucose level and in the level of multiple amino acids. Nevertheless, only the tyrosine level was shown to associate with disease-free survival among the early-stage patients, whereas no serum metabolite level is reported to significantly correlate with survival in the advanced group (39).

The importance of the metabolic supply lines can extend to more aspects of cancer biology than proliferation and metastasis, for example immune system evasion, where one mechanism concerns the indoleamine 2,3-dioxygenase 1 (IDO1)-mediated breakdown of tryptophan. Indoleamine 2,3dioxygenase 1 (IDO1) initiates tryptophan conversion in the kynurenine pathway which subsequently yields metabolites (kynurenine and others) exerting immunosuppressive effects, for example through kynurenine-aryl hydrocarbon receptor interaction leading to the induction of FoxP3+ regulatory $\mathrm{T}$ cells, which consequently compromises the antitumor response (40). Some studies furthermore show that lower serum tryptophan levels, higher kynurenine levels and a high kynurenine/tryptophan ratio associate with the development of metastases and decreased survival in different cancers (41-43).

\section{Perspectives on Targeting the Metabolic Supply Lines}

Studies seem to suggest an impact of the metabolic supply lines as these can be shown to correlate with metastatic progression and shorter survival. Table I presents an overview of a highlighted portion of the examined literature demonstrating this. Such observations could be supported by contemplating the mechanism of action in certain clinically used cancer treatments. Asparaginase, which is used as an essential tool in the treatment of acute lymphoblastic leukemia (ALL), cleaves circulatory asparagine thereby markedly reducing its concentration. ALL cells lack the ability to synthesize asparagine de novo and are therefore dependent on the circulatory supply. Thus, when the supply is eliminated, the ALL cells undergo apoptosis. Furthermore, targeting cellular metabolism in cancer in general (i.e., not specifically the supply lines) is a core element in extensively 
used chemotherapeutic treatments. Methotrexate, an antifolate interfering with for instance nucleotide synthesis, is being used in the treatment of a wide range of cancers, which at the same time illustrates how targeting metabolism supporting activities that are essential to malignancy can be broadly applied. Together, these studies in addition with previous experience of therapeutically exploiting metabolic differences in cancer show the importance for exploring the potential in strategies targeting metabolic supply lines facilitating malignancy.

However, there are some important perspectives to be considered. With regard to the amino acid perturbations, the overall changes reported by the different studies are characterized by a certain amount of diversity and discordance. Furthermore, the studies investigating amino acids generally include a relatively small number of patients which, given the complexity of the data, also presents a challenge for making more definitive conclusions. Therefore, future studies should take into consideration the number of included patients, technical issues and preanalytical factors. Concerning the actual interpretation of the results, it must be noted that the observed decreased amino acid concentrations only indirectly imply an impact of the circulatory availability of these amino acids; it indicates the utilization of the supply lines, but not directly the condition of these supply lines as to how available the given material is. It is, therefore, also not directly deducible whether lowering this availability would have therapeutic potential. It does, however, appear to be a reasonable interpretation, which would be supported by the asparaginase-example. Practically, it can furthermore be difficult to precisely determine if a given observed metabolic alteration functions as a causative factor in the disease progression or if it is simply reflecting the presence of the disease and is a consequence hereof.

Another important perspective is that engaging in developing strategies targeting the metabolic supply lines must be done with caution. This can be emphasized by the observations of unexpected responses seen in relation to antiangiogenic therapy, where the inhibition of angiogenesis may in fact provoke invasiveness as an unwanted alternative mechanism to gain vascular access (2). It is as such not impossible that a similar mechanism could apply with regard to the metabolic supply. Also, immune cells share some metabolic similarities with cancer cells $(3,9)$, and it is likewise not impossible that targeting metabolic supply lines could affect immune cell function. One supposition could then be that therapeutic potential would be achieved through securing a high supply, that is to not provoke invasive behavior and to uphold effective immune function. The lower observed amino acid levels in the studies could, in that case, be viewed to not indicate utilization but the actual availability, which would then prompt metastatic development and manifest as decreased survival. This is to emphasize that considerations like these should be made so that strategies can adapt to and counter potential problems before they arise and to maintain interpretive openness as to how strategies can be investigated most effectively.

A particularly critical aspect that must be evaluated when targeting the metabolic supply lines is that cancers are metabolically flexible, as this allows cancer cells to adapt to the given availability of metabolic building materials (44). For example, if glutamine utilization is compromised and TCA cycle function is affected, compensation by upregulation of glucose-derived anaplerosis can be seen (45). Similarly, serine can be acquired through extracellular uptake or via de novo synthesis using the glycolytic intermediate 3phosphoglycerate. Consequently, it appears that the effect of inhibiting phosphoglycerate dehydrogenase (PHGDG) as a means to target malignant serine metabolism, is dependent on the extracellular serine availability $(8,46)$. This flexibility makes it necessary to consider the possibility of targeting multiple supply lines. For example, if the glutamine supply is intervened, then glucose must to some extent be redistributed to fuel the TCA cycle, while still maintaining the task of providing enough glycolytic intermediates for branching biosynthetic and redox pathways. If the serine supply is additionally intervened, this will place further stress on the generation of glycolytic intermediates, as de novo synthesis of serine must be increased in order to adequately fuel one-carbon metabolism etc. If glucose is furthermore intervened, this would in the example constitute a compromised supply line pattern that would fail to effectively support malignant progression. This convey what is a vital consideration. Specifically, that because of the fact that generally multiple supply lines underlie malignancy and that such flexibility exists, the challenge lies in navigating the metabolic landscape in an attempt to locate the principal supply lines that may constitute patterns imperative to malignancy. This will obviously include all other metabolic building materials, not only glucose and amino acids, and since strategies can and should not target all supply lines, identifying the patterns of principal importance to cancer, which are at the same time prone to therapeutic intervention, should be the aim. Additionally, it could also be that some supply lines should be up-regulated if they facilitate critical antitumor functions.

At the same time, exploring the potential of combining such strategies with other treatment options is important. A study investigating the serum metabolic adaptations in patients receiving a PD- 1 inhibitor in three different trials found that an increasing serum kynurenine/tryptophan ratio appears as a resistance mechanism during treatment, and furthermore, that the increasing ratio is also associated with worse survival (47).

Overall, such strategies can direct the targeting of the supply lines towards the specific characteristics of a given 
cancer, or they can attempt to target, in a more general approach, the supply lines that underlie the processes fundamentally defining malignancy.

\section{Conclusion}

In conclusion, the blood circulation represents the principal supply lines delivering metabolic building materials, and augmented utilization of these supply lines facilitates the increased demands of malignant activity. Studies appear to indicate that the conditions of the supply lines can have clinical implications as they can be found to correlate with survival and the development of metastases. Intervening the supply lines could, therefore, potentially present as a strategy to limit the most fundamental components required to exert malignant functioning. This intervention should, since multiple supply lines are utilized and adaptational flexibility is exhibited, strive to target the patterns of supply lines essential to malignancy. The viability of this depends on whether there exist patterns of supply lines that are imperative to the activities that constitute malignancy, and whether there exists a therapeutic window for these patterns. Thus, the aim should be to navigate the metabolic landscape in an attempt to locate and identify the principal patterns underlying malignancy, since intervening these would, in theory, comprise a strategy that targets the thermodynamic foundation that enables the cornerstone activities essentially making a cancer a cancer.

\section{Conflicts of Interest}

The Authors declare that they have no conflicts of interest.

\section{Authors' Contributions}

OA conceived the idea for the study and drafted the manuscript. EH took part in all planning. $\mathrm{EH}$ and $\mathrm{EB}$ provided scientific expertise. OA, EH and EB edited the manuscript. All Authors have read and approved the final manuscript.

\section{References}

1 Bray F, Ferlay J, Soerjomataram I, Siegel RL, Torre LA and Jemal A: Global cancer statistics 2018: GLOBOCAN estimates of incidence and mortality worldwide for 36 cancers in 185 countries. CA Cancer J Clin 68(6): 394-424, 2018. PMID: 30207593. DOI: $10.3322 /$ caac. 21492

2 Hanahan D and Weinberg RA: Hallmarks of cancer: the next generation. Cell 144(5): 646-674, 2011. PMID: 21376230. DOI: 10.1016/j.cell.2011.02.013

3 DeBerardinis RJ and Chandel NS: Fundamentals of cancer metabolism. Sci Adv 2(5): e1600200, 2016. PMID: 27386546. DOI: $10.1126 /$ sciadv.1600200

4 Cantor JR and Sabatini DM: Cancer cell metabolism: one hallmark, many faces. Cancer Discov 2(10): 881-898, 2012. PMID: 23009760. DOI: 10.1158/2159-8290.CD-12-0345
5 Keenan MM and Chi JT: Alternative fuels for cancer cells. Cancer J 21(2): 49-55, 2015. PMID: 25815843. DOI: 10.1097/PPO.0000000000000104

6 Pavlova NN and Thompson CB: The emerging hallmarks of cancer metabolism. Cell Metab 23(1): 27-47, 2016. PMID: 26771115. DOI: 10.1016/j.cmet.2015.12.006

7 Vander Heiden MG, Cantley LC and Thompson CB: Understanding the Warburg effect: the metabolic requirements of cell proliferation. Science 324(5930): 1029-1033, 2009. PMID: 19460998. DOI: 10.1126/science.1160809

8 Choi BH and Coloff JL: The diverse functions of non-essential amino acids in cancer. Cancers (Basel) 11(5): 675, 2019. PMID: 31096630. DOI: 10.3390/cancers 11050675

9 Vander Heiden MG and DeBerardinis RJ: Understanding the intersections between metabolism and cancer biology. Cell 168(4): 657-669, 2017. PMID: 28187287. DOI: 10.1016/j.cell.2016.12.039

10 DeBerardinis RJ, Mancuso A, Daikhin E, Nissim I, Yudkoff M, Wehrli S and Thompson CB: Beyond aerobic glycolysis: transformed cells can engage in glutamine metabolism that exceeds the requirement for protein and nucleotide synthesis. Proc Natl Acad Sci USA 104(49): 19345-19350, 2007. PMID: 18032601. DOI: 10.1073/pnas.0709747104

11 Teoh ST and Lunt SY: Metabolism in cancer metastasis: bioenergetics, biosynthesis, and beyond. Wiley Interdiscip Rev Syst Biol Med 10(2), 2018. PMID: 28990735. DOI: 10.1002/wsbm.1406

12 Barua R, Templeton AJ, Seruga B, Ocana A, Amir E and Ethier JL: Hyperglycaemia and survival in solid tumours: a systematic review and meta-analysis. Clin Oncol (R Coll Radiol) 30(4): 215224, 2018. PMID: 29395413. DOI: 10.1016/j.clon.2018.01.003

13 Simon JM, Thomas F, Czernichow S, Hanon O, Lemogne C, Simon T, Pannier B and Danchin N: Hyperglycaemia is associated with cancer-related but not non-cancer-related deaths: evidence from the IPC cohort. Diabetologia 61(5): 1089-1097, 2018. PMID: 29305626. DOI: 10.1007/s00125-017-4540-8

14 Kakehi E, Kotani K, Nakamura T, Takeshima T and Kajii E: Non-diabetic glucose levels and cancer mortality: a literature review. Curr Diabetes Rev 14(5): 434-445, 2018. PMID: 28699482. DOI: 10.2174/1573399813666170711142035

15 Murtola TJ, Sälli SM, Talala K, Taari K, Tammela TLJ and Auvinen A: Blood glucose, glucose balance, and disease-specific survival after prostate cancer diagnosis in the Finnish Randomized Study of Screening for Prostate Cancer. Prostate Cancer Prostatic Dis 22(3): 453-460, 2019. PMID: 30679762. DOI: $10.1038 / \mathrm{s} 41391-018-0123-0$

16 Chen S, Tao M, Zhao L and Zhang X: The association between diabetes/hyperglycemia and the prognosis of cervical cancer patients: A systematic review and meta-analysis. Medicine (Baltimore) 96(40): e7981, 2017. PMID: 28984757. DOI: 10.1097/MD.0000000000007981

17 Hu D, Peng F, Lin X, Chen G, Liang B, Li C, Zhang H, Liao X, Lin J, Zheng $X$ and Niu W: The elevated preoperative fasting blood glucose predicts a poor prognosis in patients with esophageal squamous cell carcinoma: The Fujian prospective investigation of cancer (FIESTA) study. Oncotarget 7(40): 6524765256, 2016. PMID: 27533454. DOI: 10.18632/oncotarget.11247

18 Boursi B, Giantonio BJ, Lewis JD, Haynes K, Mamtani R and Yang YX: Serum glucose and hemoglobin A1C levels at cancer diagnosis and disease outcome. Eur J Cancer 59: 90-98, 2016. PMID: 27017290. DOI: 10.1016/j.ejca.2016.02.018 
19 Hu D, Peng F, Lin X, Zhang H, Xia Y, Lin J, Zheng X and Niu $\mathrm{W}$ : The risk trajectory between preoperative fasting glucose and common digestive tract cancer-specific mortality in the FIESTA cohort involving 6865 Chinese patients. J Cancer 10(19): 45964602, 2019. PMID: 31528223. DOI: 10.7150/jca.31184

20 Contiero P, Berrino F, Tagliabue G, Mastroianni A, Di Mauro MG, Fabiano S, Annulli M and Muti P: Fasting blood glucose and long-term prognosis of non-metastatic breast cancer: a cohort study. Breast Cancer Res Treat 138(3): 951-959, 2013. PMID: 23568483. DOI: 10.1007/s10549-013-2519-9

21 Nik-Ahd F, Howard LE, Eisenberg AT, Aronson WJ, Terris MK, Cooperberg MR, Amling CL, Kane CJ and Freedland SJ: Poorly controlled diabetes increases the risk of metastases and castration-resistant prostate cancer in men undergoing radical prostatectomy: Results from the SEARCH database. Cancer 125(16): 2861-2867, 2019. PMID: 31034601. DOI: 10.1002/ cncr.32141

22 Cui G, Zhang T, Ren F, Feng WM, Yao Y, Cui J, Zhu GL and Shi QL: High blood glucose levels correlate with tumor malignancy in colorectal cancer patients. Med Sci Monit 21: 3825-3833, 2015. PMID: 26644185. DOI: 10.12659/msm.894783

23 Payen VL, Porporato PE, Baselet B and Sonveaux P: Metabolic changes associated with tumor metastasis, part 1: tumor $\mathrm{pH}$, glycolysis and the pentose phosphate pathway. Cell Mol Life Sci 73(7): 1333-1348, 2016. PMID: 26626411. DOI: 10.1007/s00018015-2098-5

$24 \mathrm{Bi} \mathrm{X}$ and Henry CJ: Plasma-free amino acid profiles are predictors of cancer and diabetes development. Nutr Diabetes 7(3): e249, 2017. PMID: 28287627. DOI: 10.1038/nutd.2016.55

25 Berker Y, Vandergrift LA, Wagner I, Su L, Kurth J, Schuler A, Dinges SS, Habbel P, Nowak J, Mark E, Aryee MJ, Christiani DC and Cheng LL: Magnetic resonance spectroscopy-based metabolomic biomarkers for typing, staging, and survival estimation of early-stage human lung cancer. Sci Rep 9(1): 10319, 2019. PMID: 31311965. DOI: 10.1038/s41598-01946643-5

26 Ling HH, Pan YP, Fan CW, Tseng WK, Huang JS, Wu TH, Chou WC, Wang $\mathrm{CH}$, Yeh KY and Chang PH: Clinical significance of serum glutamine level in patients with colorectal cancer. Nutrients 11(4): 898, 2019. PMID: 31010101. DOI: 10.3390/nu11040898

27 Lieu EL, Nguyen T, Rhyne S and Kim J: Amino acids in cancer. Exp Mol Med 52(1): 15-30, 2020. PMID: 31980738. DOI: 10.1038/s12276-020-0375-3

$28 \mathrm{Ma} \mathrm{H}$, Hasim A, Mamtimin B, Kong B, Zhang HP and Sheyhidin I: Plasma free amino acid profiling of esophageal cancer using high-performance liquid chromatography spectroscopy. World J Gastroenterol 20(26): 8653-8659, 2014 PMID: 25024622. DOI: 10.3748/wjg.v20.i26.8653

29 Newman AC and Maddocks ODK: One-carbon metabolism in cancer. $\mathrm{Br} \mathrm{J}$ Cancer 116(12): 1499-1504, 2017. PMID: 28472819. DOI: $10.1038 /$ bjc. 2017.118

30 Shen J, Song R, Hodges TR, Heimberger AB and Zhao H: Identification of metabolites in plasma for predicting survival in glioblastoma. Mol Carcinog 57(8): 1078-1084, 2018. PMID: 29603794. DOI: $10.1002 / \mathrm{mc} .22815$

31 Vsiansky V, Svobodova M, Gumulec J, Cernei N, Sterbova D, Zitka O, Kostrica R, Smilek P, Plzak J, Betka J, Kalfert D, Masarik M and Raudenska M: Prognostic significance of serum free amino acids in head and neck cancers. Cells 8(5): 428, 2019. PMID: 31075822. DOI: 10.3390/cells 8050428
32 Jobard E, Pontoizeau C, Blaise BJ, Bachelot T, Elena-Herrmann $\mathrm{B}$ and Trédan O: A serum nuclear magnetic resonance-based metabolomic signature of advanced metastatic human breast cancer. Cancer Lett 343(1): 33-41, 2014. PMID: 24041867. DOI: 10.1016/j.canlet.2013.09.011

33 Shen CT, Zhang Y, Liu YM, Yin S, Zhang XY, Wei WJ, Sun ZK, Song HJ, Qiu ZL, Wang CR and Luo QY: A distinct serum metabolic signature of distant metastatic papillary thyroid carcinoma. Clin Endocrinol (Oxf) 87(6): 844-852, 2017. PMID: 28755525. DOI: $10.1111 /$ cen.13437

34 Zheng H, Dong B, Ning J, Shao X, Zhao L, Jiang Q, Ji H, Cai A, Xue $\mathrm{W}$ and Gao H: NMR-based metabolomics analysis identifies discriminatory metabolic disturbances in tissue and biofluid samples for progressive prostate cancer. Clin Chim Acta 501: 241251, 2020. PMID: 31758937. DOI: 10.1016/j.cca.2019.10.046

35 Graboń W, Mielczarek-Puta M, Chrzanowska A and BarańczykKuźma A: L-arginine as a factor increasing arginase significance in diagnosis of primary and metastatic colorectal cancer. Clin Biochem 42(4-5): 353-357, 2009. PMID: 19101531. DOI: 10.1016/j.clinbiochem.2008.11.018

36 Gao H, Dong B, Liu X, Xuan H, Huang Y and Lin D: Metabonomic profiling of renal cell carcinoma: high-resolution proton nuclear magnetic resonance spectroscopy of human serum with multivariate data analysis. Anal Chim Acta 624(2): 269-277, 2008. PMID: 18706333. DOI: 10.1016/j.aca.2008.06.051

37 Jin H, Qiao F, Chen L, Lu C, Xu L and Gao X: Serum metabolomic signatures of lymph node metastasis of esophageal squamous cell carcinoma. J Proteome Res 13(9): 4091-4103, 2014. PMID: 25162382. DOI: 10.1021/pr500483z

38 Schafer ZT, Grassian AR, Song L, Jiang Z, Gerhart-Hines Z, Irie HY, Gao S, Puigserver P and Brugge JS: Antioxidant and oncogene rescue of metabolic defects caused by loss of matrix attachment. Nature 461(7260): 109-113, 2009. PMID: 19693011. DOI: $10.1038 /$ nature 08268

39 Casadei-Gardini A, Del Coco L, Marisi G, Conti F, Rovesti G, Ulivi P, Canale M, Frassineti GL, Foschi FG, Longo S, Fanizzi FP and Giudetti AM: ${ }^{1} \mathrm{H}-\mathrm{NMR}$ based serum metabolomics highlights different specific biomarkers between early and advanced hepatocellular carcinoma stages. Cancers (Basel) 12(1): 241, 2020. PMID: 31963766. DOI: 10.3390/cancers 12010241

40 Zhai L, Spranger S, Binder DC, Gritsina G, Lauing KL, Giles FJ and Wainwright DA: Molecular pathways: Targeting IDO1 and other tryptophan dioxygenases for cancer immunotherapy. Clin Cancer Res 21(24): 5427-5433, 2015. PMID: 26519060. DOI: $10.1158 / 1078-0432 . C C R-15-0420$

41 Masaki A, Ishida T, Maeda Y, Ito A, Suzuki S, Narita T, Kinoshita S, Takino H, Yoshida T, Ri M, Kusumoto S, Komatsu H, Inagaki H, Ueda R, Choi I, Suehiro Y and Iida S: Clinical significance of tryptophan catabolism in Hodgkin lymphoma. Cancer Sci 109(1): 74-83, 2018. PMID: 29080383. DOI: $10.1111 /$ cas. 13432

42 Ferns DM, Kema IP, Buist MR, Nijman HW, Kenter GG and Jordanova ES: Indoleamine-2,3-dioxygenase (IDO) metabolic activity is detrimental for cervical cancer patient survival. Oncoimmunology 4(2): e981457, 2015. PMID: 25949879. DOI: 10.4161/2162402X.2014.981457

43 Weinlich G, Murr C, Richardsen L, Winkler C and Fuchs D: Decreased serum tryptophan concentration predicts poor prognosis in malignant melanoma patients. Dermatology 214(1): 8-14, 2007. PMID: 17191041. DOI: 10.1159/000096906 
44 Boroughs LK and DeBerardinis RJ: Metabolic pathways promoting cancer cell survival and growth. Nat Cell Biol 17(4): 351-359, 2015. PMID: 25774832. DOI: 10.1038/ncb3124

45 Cheng T, Sudderth J, Yang C, Mullen AR, Jin ES, Matés JM and DeBerardinis RJ: Pyruvate carboxylase is required for glutamine-independent growth of tumor cells. Proc Natl Acad Sci USA 108(21): 8674-8679, 2011. PMID: 21555572. DOI: 10.1073/pnas.1016627108

46 Sullivan MR, Mattaini KR, Dennstedt EA, Nguyen AA, Sivanand S, Reilly MF, Meeth K, Muir A, Darnell AM, Bosenberg MW, Lewis CA and Vander Heiden MG: Increased serine synthesis provides an advantage for tumors arising in tissues where serine levels are limiting. Cell Metab 29(6): 1410-1421.e4, 2019. PMID: 30905671. DOI: 10.1016/j.cmet.2019.02.015
47 Li H, Bullock K, Gurjao C, Braun D, Shukla SA, Bossé D, Lalani AA, Gopal S, Jin C, Horak C, Wind-Rotolo M, Signoretti S, McDermott DF, Freeman GJ, Van Allen EM, Schreiber SL, Stephen Hodi F, Sellers WR, Garraway LA, Clish CB, Choueiri TK and Giannakis M: Metabolomic adaptations and correlates of survival to immune checkpoint blockade. Nat Commun 10(1): 4346, 2019. PMID: 31554815. DOI: 10.1038/s41467-019-12361-9

Received March 17, 2021

Revised May 4, 2021 Accepted May 10, 2021 